\title{
Holographic melting and related properties of mesons in a quark-gluon plasma
}

\author{
Kasper Peeters* and Marija Zamaklar ${ }^{\dagger}$ \\ MPI für Gravitationsphysik, Am Mühlenberg 1, 14476 Golm, Germany \\ Jacob Sonnenschein \\ School of Physics and Astronomy, The Raymond and Beverly Sackler Faculty of Exact Sciences, Tel Aviv University, \\ Ramat Aviv, 69978, Israel
}

(Received 18 July 2006; published 17 November 2006)

\begin{abstract}
We analyze mesons at finite temperature in a chiral, confining string dual. The temperature dependence of low-spin as well as high-spin meson masses is shown to exhibit a pattern familiar from the lattice. Furthermore, we find the dissociation temperature of mesons as a function of their spin, showing that at a fixed quark mass, mesons with larger spins dissociate at lower temperatures. The Goldstone bosons associated with chiral symmetry breaking are shown to disappear above the chiral symmetry restoration temperature. Finally, we show that holographic considerations imply that large-spin mesons do not experience drag effects when moving through the quark-gluon plasma. They do, however, have a maximum velocity for fixed spin, beyond which they dissociate.
\end{abstract}

DOI: 10.1103/PhysRevD.74.106008

PACS numbers: 11.25.Tq, 12.38.Mh, 11.25.- w

\section{INTRODUCTION AND SUMMARY}

Of the various gravity duals to confining, nonsupersymmetric gauge theories in four dimensions, the SakaiSugimoto model [1,2] stands out because it contains chiral fermions as well as an associated chiral symmetry breaking transition. This model has been analyzed in quite some detail at zero temperature. More recent work has analyzed the phase diagram of the model, showing an interesting structure in which the confinement/deconfinement transition does not necessarily coincide with the chiral symmetry breaking transition [3] (see also [4]). In the present paper, we will analyze the various phases which were found in [3] in more detail, focussing on the properties of mesons.

The D4-brane background of the Sakai-Sugimoto model with Euclidean signature exhibits two circle directions. The analysis of [3] has shown that this geometrical structure, plus the presence of the D8-probes, leads to three different phases (when the ratio $L / R$, see Fig. 1, is sufficiently small). In the low-temperature phase the background is the Euclidean continuation of a Lorentzian background without any horizon. Gluons are confined in this phase. After the confinement/deconfinement transition for the gluons, we enter the intermediate-temperature phase, in which the Euclidean geometry is instead given by the continuation of a black brane geometry. In this phase gluons are deconfined, but chiral symmetry is still broken. Mesonic bound states still exist, as the D8-brane embedding is not yet touching the horizon. At sufficiently high temperature, the lowest-energy configuration of the D8branes is one in which they hang vertically from infinity down to the horizon. This is the high-temperature phase, in

\footnotetext{
*Email: kasper.peeters@aei.mpg.de

${ }^{\dagger}$ Email: cobi@post.tau.ac.il

Email: marija.zamaklar@aei.mpg.de
}

which chiral symmetry is restored. If the ratio $L / R>0.97$, there is no intermediate-temperature phase, so that the confinement/deconfinement and the chiral symmetry breaking transition coincide. For more details, we refer to [3].

In the present paper, we will have a close look at the spectrum of low-spin as well as high-spin mesons in these three phases. From both lattice results and experiment, it is known that the meson spectrum shows interesting behavior as the temperature goes up. There is abundant evidence, for instance, that heavy-quark bound states can survive in a quark-gluon plasma to temperatures which are substantially higher than the confinement/deconfinement transition [5]. Moreover, heavy-quark mesons tend to survive to higher temperatures than light-quark mesons (see e.g. $[6,7])$. We would like to see to which extent the SakaiSugimoto model is able to say something qualitatively about such situations.

Without further ado, let us briefly summarize the outcome of our analysis. Firstly, we find that the temperature dependence of both small-spin (Fig. 4) and large-spin (Fig. 12) mesons is qualitatively similar to what is known from mesons in a quark-gluon plasma [8-10]. That is, the masses of mesons with low excitation number go down as the temperature goes up. The $\rho$ and $a_{1}$ meson masses approach each other, although they do not become degenerate at the temperature where chiral symmetry is restored. For higher excitation level, the temperature dependence appears to be more complicated (Fig. 5).

Secondly, we analyze the temperature dependence of large-spin mesons, which are modeled by rotating $U$-shaped strings hanging from the probe brane. We find that for large-spin mesons there is a maximum value of angular momentum beyond which mesons cannot exist and have to "melt", i.e. dissociate (Fig. 11). For a given spin, the maximum temperature can be substantially lower than 


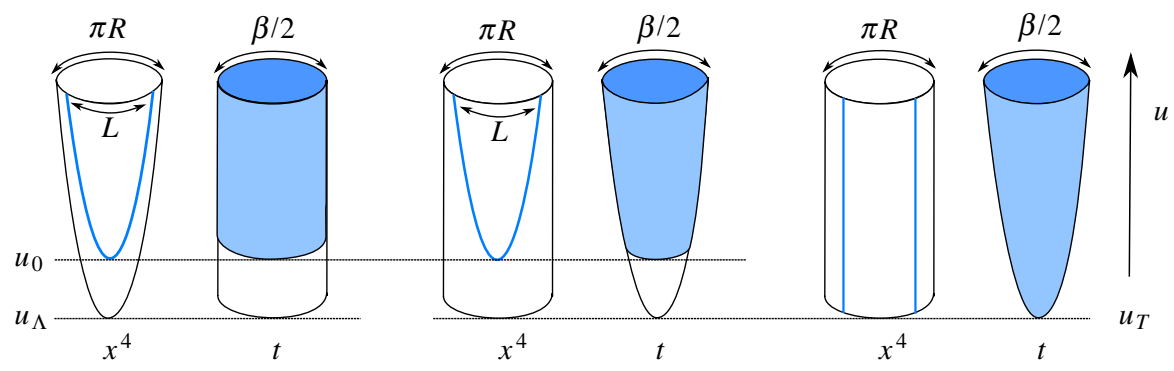

FIG. 1 (color online). The Sakai \& Sugimoto model in the low-temperature (left panel), intermediate-temperature (middle panel) and high-temperature (right panel) phases. Depicted are the two compact Euclidean directions $x^{4}$ (the Kaluza-Klein circle) and $t$ (Wick rotated time). The D8 embedding is shown as well (in blue).

the temperature of the chiral symmetry restoration transition. We consider this maximum-spin effect a prediction of the string/gauge-based models, as it is quite generic and qualitatively independent of the specifics of the background.

Thirdly, we find that large-spin mesons do not experience a drag force. Instead, there is a critical velocity beyond which a fixed-spin meson will dissociate. Equivalently, the maximum interquark distance goes down as the velocity is increased (Fig. 16). The absence of drag is unlike the situation for quark probes in a quarkgluon plasma [11-17]. For large-spin mesons at finite temperature, one can find generalized solutions where the meson moves with linear velocity, rigidly, with free boundary conditions in the direction of motion (Fig. 15). Hence one does not need to apply any force to maintain this motion. In the dual language, this is a reflection of the fact that if the quark-gluon plasma is not hot enough to dissociate mesons, then these color singlets will not experience a drag force generated by monopole interactions with the medium. ${ }^{1}$

\section{REVIEW OF THE SYSTEM AT VARIOUS TEMPERATURES}

In this section we review the various phases of the SakaiSugimoto model, originally proposed in [1,2] and analyzed at finite-temperature in [3]. Let us first recall the Euclideanized model at zero temperature. The pure glue theory is described by a background with a nontrivial metric,

$$
\begin{aligned}
d s_{\mathrm{con}}^{2}= & \left(\frac{u}{R_{\mathrm{D} 4}}\right)^{3 / 2}\left[\mathrm{~d} t 2+\delta_{i j} \mathrm{~d} x^{i} \mathrm{~d} x^{j}+f_{\Lambda}(u)\left(\mathrm{d} x^{4}\right)^{2}\right] \\
& +\left(\frac{R_{\mathrm{D} 4}}{u}\right)^{3 / 2}\left[\frac{d u^{2}}{f_{\Lambda}(u)}+u^{2} d \Omega_{4}\right],
\end{aligned}
$$

as well as a nonconstant dilaton and a fourform RR field

\footnotetext{
${ }^{1}$ The interaction with the medium could be still via dipole (or higher moment) interactions. These, however, require coupling of the string sigma model to the fields on the world volume of the brane, and will be discussed elsewhere.
}

strength given by

$$
\begin{aligned}
& e^{\phi}=g_{s}\left(\frac{u}{R_{\mathrm{D} 4}}\right)^{3 / 4}, \quad F_{4}=\frac{2 \pi N_{c}}{V_{4}} \epsilon_{4}, \\
& R_{\mathrm{D} 4}^{3}=\pi g_{s} N_{c} l_{s}^{3}, \quad f_{\Lambda}(u):=1-\left(\frac{u_{\Lambda}}{u}\right)^{3} .
\end{aligned}
$$

Here $u$ is the radial direction, which is bounded from below by $u \geq u_{\Lambda}$. Our four-dimensional world is along the $t$ and $x^{i}(i=1,2,3)$ directions. The main characteristic feature of the background is the submanifold spanned by $x^{4}$ and $u$, which has the geometry of a cigar. The tip of the cigar is nonsingular if and only if the periodicity of $x^{4}$ is

$$
\delta x^{4}=\frac{4 \pi}{3}\left(\frac{R_{\mathrm{D} 4}^{3}}{u_{\Lambda}}\right)^{1 / 2}=: 2 \pi R .
$$

Therefore, the circle $x^{4}$ shrinks from its asymptotic size $2 \pi R$ down to zero and smoothly caps off at $u=u_{\Lambda}$. Chiral quarks in the fundamental representation are incorporated by introducing a probe D8-brane into the system. The probe fills out the full space, except in the submanifold of the cigar, where it has a nontrivial profile. At zero temperature, the spectrum of the glueballs (obtained from the fluctuations of the supergravity fields of the background), as well as of low-spin mesons (obtained from the fluctuations of the fields on the probe brane) is discrete, signalling that we are in the confining phase.

Turning on the temperature $T$ corresponds to the compactification of the Euclidean time direction $t$. The temperature is related to the size $\beta$ of the compact time direction through the standard field theory relation $\beta=$ $1 / T$. We now have two circle directions, one being the asymptotic circle of the cigar discussed above, with period $2 \pi R$, and the other one being the thermal circle, with period $\beta$. This geometry is depicted in the left panel of Fig. 1, which also shows the embedding of the D8-brane. The glueball and meson spectra in this phase are still discrete. In fact, they are unmodified with respect to the zero-temperature situation, given that the metric is unmodified (modulo the global identification). We will refer to this phase as the low-temperature phase. 
As the temperature of the system is increased, the system will at a certain point undergo a first-order phase transition in which the gluonic degrees of freedom get deconfined. This is reflected in the change of the background metric from (1) to

$$
\begin{aligned}
\mathrm{d} s_{\text {decon }}^{2}= & \left(\frac{u}{R_{\mathrm{D} 4}}\right)^{3 / 2}\left[f_{T}(u) \mathrm{d} t^{2}+\delta_{i j} \mathrm{~d} x^{i} \mathrm{~d} x^{j}+\left(\mathrm{d} x^{4}\right)^{2}\right] \\
& +\left(\frac{R_{\mathrm{D} 4}}{u}\right)^{3 / 2}\left[\frac{\mathrm{d} u^{2}}{f_{T}(u)}+u^{2} \mathrm{~d} \Omega_{4}^{2}\right]
\end{aligned}
$$

with $f_{T}(u):=1-\left(\frac{u_{T}}{u}\right)^{3}$.

The metric has the same form as the low-temperature metric (1), but with the role of the $t$ and $x^{4}$ directions exchanged. This is depicted in the middle and right panels of Fig. 1. The phase transition between the metric (1) and the metric (4) happens when their free energies are equal, which is when the two circles have the same radius, i.e. when $T=T_{c}=1 / 2 \pi R$. This transition is first-order because the solutions do not smoothly connect, and continue to exist as separate solutions both below and above the transition. The spectrum of the glueball fluctuations in the phase described by (4) is continuous, signalling the deconfinement of the gluonic degrees of freedom.

An order parameter of this phase transition is the Polyakov loop, corresponding to a string wrapped around the time direction. In the low-temperature phase the time circle is noncontractible and not the boundary of a disc, so that the Polyakov loop vanishes. After the transition, this circle becomes contractible, resulting in a nonzero expectation value for the Polyakov loop. Other order parameters are the Wilson loop (which has a linear quark/antiquark potential in the first background but vanishing tension in the second) and the behavior of the free energy as a function of $N_{c}$ (namely $\sim N_{c}^{0}$ for the first background and $\sim N_{c}^{2}$ for the second).

In contrast to the gluonic degrees of freedom, the matter degrees of freedom may still have bound states in this phase. In order to analyze the matter phases, one needs to determine the shape of the D8-brane, which can be obtained from the DBI part of the effective action,

$$
S_{\mathrm{DBI}}=T_{8} \int \mathrm{d} t \mathrm{~d}^{3} x \mathrm{~d} x^{4} \mathrm{~d} \Omega_{4} e^{-\phi} \sqrt{-\operatorname{det} \hat{g}} .
$$

In the two backgrounds (1) and (4), the DBI actions have the following form,

$$
\begin{aligned}
S_{\mathrm{DBI}}^{\mathrm{con}} & =\frac{\hat{T}_{8}}{g_{s}} \int \mathrm{d} x^{4} u^{4} \sqrt{f(u)+\frac{R_{D 4}^{3}}{u^{3}} \frac{u^{\prime 2}}{f(u)^{\prime 2}}}, \\
S_{\mathrm{DBI}}^{\mathrm{decon}} & =\frac{\hat{T}_{8}}{g_{s}} \int \mathrm{d} x^{4} u^{4} \sqrt{f(u)+\frac{R_{D 4}^{3}}{u^{3}} u^{\prime 2}},
\end{aligned}
$$

where from now on we will drop the subscript on $f$ as it is clear from the context which one should be used. In the confining background, the solution to the equations of motion (depicted by the blue curve on the left panel of Fig. 1) is given by

$$
x^{4}(u)=u_{0}^{4} f\left(u_{0}\right)^{1 / 2} \int_{u_{0}}^{u} \frac{\mathrm{d} u}{\left(\frac{u}{R_{\mathrm{D} 4}}\right)^{3 / 2} f(u) \sqrt{u^{8} f(u)-u_{0}^{8} f\left(u_{0}\right)}} .
$$

The D8-brane solution in the middle panel is related to this one by the simple expression

$$
\frac{\mathrm{d} x_{\text {decon }}^{4}}{\mathrm{~d} u}=\sqrt{f(u)} \frac{\mathrm{d} x_{\text {con }}^{4}}{\mathrm{~d} u} .
$$

However, there is a second configuration in the deconfined background, in which the embedding is simply given by $x^{4}=$ const (depicted in the third panel of Fig. 1). The most important fact about this third phase is that chiral symmetry is restored. Because there are two stacks of branes, every mode will appear twice, as a representation of $\mathrm{U}\left(N_{f}\right)_{\mathrm{L}}$ and of $\mathrm{U}\left(N_{f}\right)_{\mathrm{R}}$. So we potentially have two phases in the deconfined background, which we will call the intermediate- and high-temperature phase. Whether or not the intermediate-temperature phase is realized depends on the ratio $L / R$, where $L$ is the asymptotic distance between the D8 and anti-D8 (see Fig. 1). In [3] it was found that for $L / R>0.97$, the intermediate phase is absent altogether, i.e. the confinement/deconfinement and chiral symmetry restoration transitions occur simultaneously. One can map the parameter $L / R$ to a gauge dynamical parameter by noting that $1 / R$ is the scale of the glueball masses, while (as we will show in Sec. III), $1 / L$ is proportional to the mass of mesons composed of quarks of large constituent mass, $u_{0} \gg u_{T}$. We refer the reader to [3] for more details on the full phase diagram, including the region where the supergravity picture is not valid.

In the string/gauge duality models, one associates lowspin mesons to fluctuations of the gauge fields and (pseudo) scalars that live on the probe branes, while high-spin ones are associated to spinning string configurations. We will address the thermal spectrum of the former in Sec. III and the latter in Sec. IV.

\section{LOW-SPIN MESONS AT INTERMEDIATE TEMPERATURE}

In this section we will analyze the spectrum of low-spin mesons (with spin $\leq 1$ ) in the intermediate-temperature regime. The spectrum of low-spin mesons in the lowtemperature phase is unmodified with respect to zero temperature. This is a consequence of the fact that the (Euclidean) metric is globally unmodified (the only difference is that there is now a global periodic identification of the Euclidean time direction). This perhaps unexpected feature seems to be a generic property of large- $N_{c}$ theories $[3,18,19]$ (see also the subsection of Sec. III). 
In the intermediate-temperature phase we expect that the spectrum of low-spin mesons is discrete, because the probe does not intersect the horizon. This is similar to the mechanism which ensures discreteness of the spectrum at zero temperature. Furthermore, given that the effective tension of strings near the brane decreases with the increase of temperature, one expects that the masses of the mesons decrease as the probe brane comes closer to the horizon, or equivalently, as the temperature is increased. Indeed, our explicit computation shows this behavior.

Our starting point is the metric of the background describing the hot gluonic plasma, as given in (4). This background leads to an induced metric on the D8-brane world volume, which reads

$$
\begin{aligned}
d \hat{s}_{\text {interm }}^{2}= & \left(\frac{u}{R_{\mathrm{D} 4}}\right)^{3 / 2}\left(f(u) \mathrm{d} t^{2}+\delta_{i j} \mathrm{~d} x^{i} \mathrm{~d} x^{j}\right) \\
& +\left[\left(\frac{R_{\mathrm{D} 4}}{u}\right)^{3 / 2} \frac{1}{f(u)}+\left(\frac{\mathrm{d} x^{4}}{\mathrm{~d} u}\right)^{2}\left(\frac{u}{R_{\mathrm{D} 4}}\right)^{3 / 2}\right] \mathrm{d} u^{2} \\
& +\left(\frac{R_{\mathrm{D} 4}}{u}\right)^{3 / 2} u^{2} \mathrm{~d} \Omega_{4}^{2} .
\end{aligned}
$$

We are interested in computing the spectrum of vector mesons, by considering small fluctuations on the worldvolume gauge fields of the probe D8-brane (scalar and pseudoscalar mesons can be treated with similar methods but will not be discussed here). In order to do this, we first expand the gauge field on the world volume of the D8brane as [1]

$$
\begin{aligned}
F_{\mu \nu} & =\sum_{n} F_{\mu \nu}^{(n)}\left(x^{\rho}\right) \psi_{(n)}(u), \\
F_{\mu u} & =\sum_{n} \partial_{\mu} \varphi^{(n)} \phi_{(n)}(u)-B_{\mu}^{(n)} \partial_{u} \psi_{(n)}(u) \\
& =\partial_{\mu} \varphi^{(0)} \phi_{0}+\sum_{n \geq 1}\left(\partial_{\mu} \varphi^{(n)}-B_{\mu}^{(n)}\right) \partial_{u} \psi_{(n)} .
\end{aligned}
$$

where the last line is obtained by taking $\phi_{(n)}=$ $m_{n}^{-1} \partial_{u} \psi_{(n)}(u)$. To simplify the consideration, we choose to focus on the spacelike components $B_{i}$ of the vector fields. Moreover, we only consider the masses defined by

$$
\partial_{0}^{2} B_{i}^{(n)}=-m_{n}^{2} B_{i}^{(n)},
$$

that is, we consider the behavior of the pole mass rather than the screening mass. Thus, we consider only spatially homogeneous modes, i.e. we consider the equation of motion for fields satisfying $\partial_{i} B_{j}^{(n)}=0 .{ }^{2}$ In this case, after using the equations explicit probe-brane embedding (7) and (8) the probe-brane action reduces to

\footnotetext{
${ }^{2}$ For nonhomogeneous modes the definition of mass becomes more subtle. However, since we expect that the homogeneous modes considered here are rich enough to demonstrate all relevant features of the spectrum as a function of the temperature, we restrict our attention to this case.
}

$$
\begin{aligned}
\hat{S}_{\text {trunc }}= & \int \mathrm{d}^{4} x \mathrm{~d} u u^{4} \gamma^{1 / 2}\left(\frac{R_{\mathrm{D} 4}}{u}\right)^{3 / 2} f(u)^{1 / 2} \\
& \times\left[\frac{1}{\gamma f(u)}\left(\partial_{0} \varphi^{(0)}\right)^{2} \phi^{(0)} \phi^{(0)}\right. \\
& -\frac{1}{f(u)}\left(\frac{R_{\mathrm{D} 4}}{u}\right)^{3} \partial_{0} B_{i}^{(m)} \partial_{0} B_{(n)}^{i} \psi_{(m)} \psi_{(n)} \\
& \left.+\gamma^{-1} B_{i}^{(m)} B_{(n)}^{i} \partial_{u} \psi_{(m)} \partial_{u} \psi_{(n)}\right]
\end{aligned}
$$

with $\quad \gamma \equiv \frac{u^{8}}{u^{8} f(u)-u_{0}^{8} f\left(u_{0}\right)}$.

After a partial integration with respect to the $u$-coordinate, the equation of motion for the field $B_{i}^{(m)}$ becomes

$$
\begin{aligned}
& \frac{u^{4} \gamma^{1 / 2}}{f(u)^{1 / 2}}\left(\frac{R_{\mathrm{D} 4}}{u}\right)^{9 / 2} \partial_{0}^{2} B_{i}^{(n)} \psi_{(n)} \\
& \quad-\partial_{u}\left(u^{4} \gamma^{-1 / 2} f(u)^{1 / 2}\left(\frac{R_{\mathrm{D} 4}}{u}\right)^{3 / 2} \partial_{u} \psi_{(n)}\right) B_{i}^{(n)}=0 .
\end{aligned}
$$

This equation will reduce to the canonical form (with thermal masses $m_{n}$ as in (11)) if the modes $\psi_{(n)}$ satisfy the equation

$$
\begin{aligned}
- & u^{1 / 2} \gamma^{-1 / 2} f(u)^{1 / 2} \partial_{u}\left(u^{5 / 2} \gamma^{-1 / 2} f(u)^{1 / 2} \partial_{u} \psi_{(n)}\right) \\
& =R_{\mathrm{D} 4}^{3} 3 m_{n}^{2} \psi_{(n)} .
\end{aligned}
$$

Equation (14) is very similar to the equation in the zerotemperature case. The only difference is the appearance of extra factors $f(u)^{1 / 2}$ in the term on the left-hand side. In order to have canonically normalized kinetic terms, the modes should also satisfy the normalization conditions

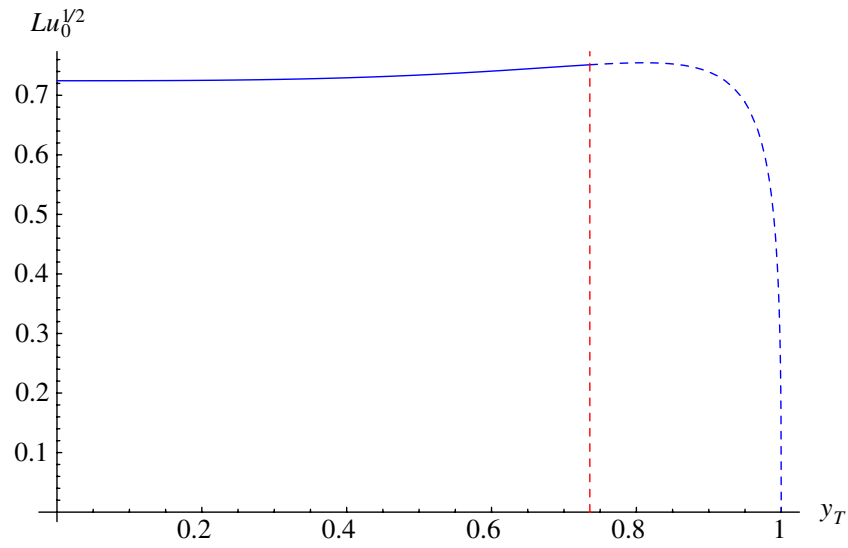

FIG. 2 (color online). The asymptotic distance $L$ between the D8 and anti-D8 as a function of $y_{T}:=u_{T} / u_{0}$. The red dashed line indicates the phase transition to the high-temperature phase, above which one should instead use two parallel D8 stacks. Note that " $L=$ const $\rightarrow u_{0}=$ const" holds to good approximation in the intermediate-temperature regime. 

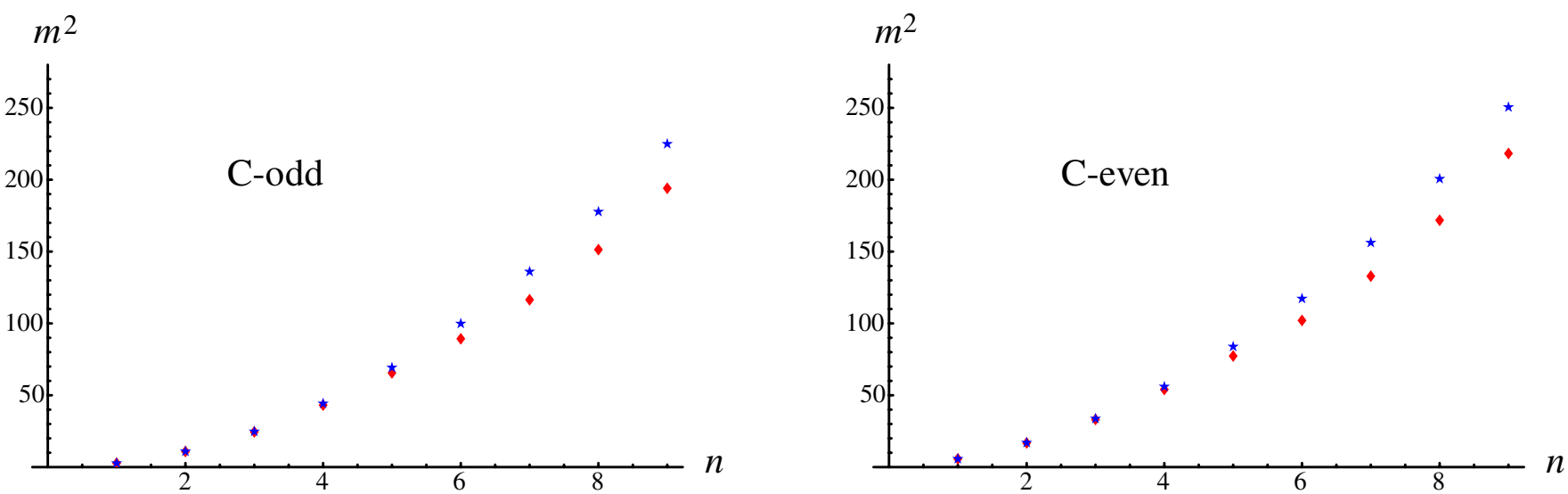

FIG. 3 (color online). Meson masses (squared) as a function of level $n$ for the zero temperature (upper dots) and intermediatetemperature phases (lower dots), for an asymptotic separation between the D8 and anti-D8 fixed at $L / R=0.63$, with $u_{\Lambda}=u_{T}=1$. (Extensions of these curves to even higher level show $m^{2} \sim n^{2}$ behavior for large $n$, both at zero and at finite temperature, which is one of the unrealistic features of these models [36-38]).

$$
\begin{aligned}
& \int_{u_{0}}^{\infty} \mathrm{d} u u^{4} \gamma^{1 / 2} f^{-1 / 2}(u)\left(\frac{u}{R_{\mathrm{D} 4}}\right)^{9 / 2} \psi_{(m)} \psi_{(n)}=\delta_{m n}, \\
& \int_{u_{0}}^{\infty} \mathrm{d} u u^{4} \gamma^{-1 / 2} f^{-1 / 2}(u)\left(\frac{R_{\mathrm{D} 4}}{u}\right)^{3 / 2} \phi^{(0)} \phi^{(0)}=1 \text {. }
\end{aligned}
$$

The zero mode $\phi^{(0)}=u^{-5 / 2} f(u)^{-1 / 2} \gamma^{1 / 2}$ is normalizable with this norm (there is no problem at the horizon because $u_{0}>u_{T}$ ), and so we see that there is still a massless pion present in the intermediate-temperature phase.

Before applying numerical methods to compute the spectrum, it is interesting to note that in the limit of $u_{0} \gg$ $u_{T}$ the spectrum simplifies and one can easily determine the scale of the meson masses. In this limit, which corresponds to a small separation distance between the stacks of branes and antibranes $L \ll R$, the thermal factor $f(u) \rightarrow 1$ and, in particular, also $f\left(u_{0}\right) \rightarrow 1$. Therefore,

$$
\gamma \equiv \frac{u^{8}}{u^{8} f(u)-u_{0}^{8} f\left(u_{0}\right)} \rightarrow \frac{1}{1-y^{-8}}
$$

where the dimensionless quantity $y \equiv u / u_{0}$. In fact, for this case we can rewrite (14) in terms of $y$ in the following form

$$
-y^{1 / 2} \gamma^{-1 / 2}(y) \partial_{y}\left(y^{5 / 2} \gamma^{-1 / 2}(y) \partial_{y} \psi_{(n)}\right)=\frac{R_{\mathrm{D} 4}^{3}}{u_{0}} m_{n}^{2} \psi_{(n)} .
$$

Now since the left-hand side is expressed in terms of the dimensionless quantity $y$, the right-hand side should also be dimensionless which implies that

$$
m_{n}^{2} \sim \frac{u_{0}}{R_{\mathrm{D} 4}^{3}}
$$

Now since $u_{0} \sim 1 / L^{2}$ (see below), the final conclusion is that the mass of these "short" mesons scales as

$$
M_{\text {meson }} \sim \frac{1}{L} .
$$

To find the remainder of the spectrum, we can solve Eq. (14) numerically using a shooting technique. We will keep the asymptotic separation $L$ between the D8 and antiD8 stacks fixed, and use the expression

$$
L=\int \mathrm{d} x^{4}=\int_{u_{0}}^{\infty} \frac{\mathrm{d} u}{u^{\prime}}
$$

to determine $u_{0}$ for a given temperature (see also Fig. 2). The results for the thermal masses $m_{n}^{2}$ of the vector mesons are depicted in Fig. 3. The spectrum is discrete, labeled by the mode number $n$ and parity. By comparing with the zero-temperature result, we observe that the masses of light mesons decrease as the temperature is increased. The temperature dependence of the masses of the " $\rho$ " and " $a_{1}$ " mesons are shown in Fig. 4.

Our findings are in qualitative agreement with lattice computations $[8,20]$ and the results of the "phenomenological" model of Karch et al. [21], although we do not see the degeneracy of the $\rho$ and $a_{1}$ meson masses at high temperature. Moreover, we will see in Sec. V that the spectrum becomes continuous in the phase where chiral symmetry is restored. Therefore, the phase transition from intermediate to high temperature, which is first-order in our model, is rather different from QCD. However, what we see is consistent with the fact that mesons in the chirally symmetric phase are extremely unstable in the large- $N_{c}$ limit: their decay widths scale as a positive power of $N_{c}{ }^{3}$.

This qualitative behavior of the spectrum in our model (and the ad-hoc model of [21]) is a direct consequence of the fact that the flavour branes are, for fixed $L$, closer to the

\footnotetext{
${ }^{3}$ For results of other, nonholographic models see e.g. the summary in [22].
} 


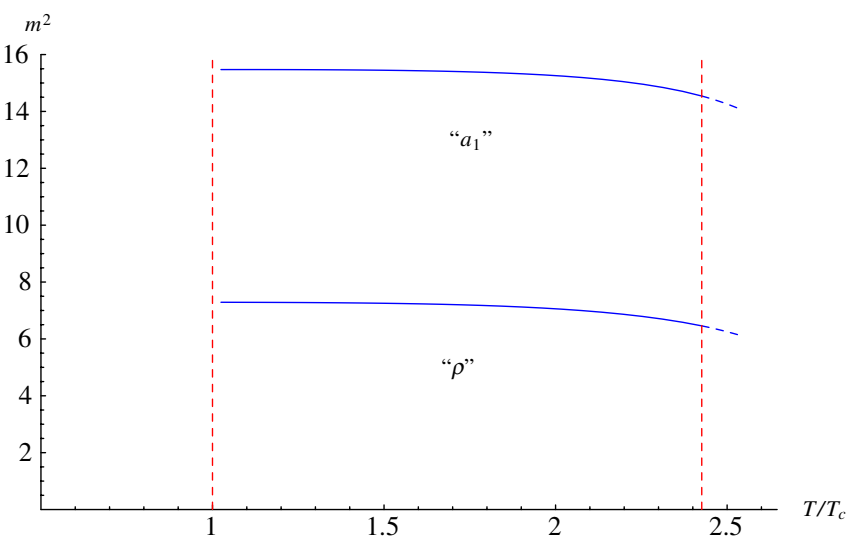

FIG. 4 (color online). Squared masses of the two lightest vector mesons as a function of temperature in the intermediate-temperature regime, for $L=0.256<0.97 R$. Masses are normalized as in (14). The decrease of the mass is in qualitative agreement with results from other models and experiments [9], though one should be cautious since the latter are not in the large- $N_{c}$ limit. The $\rho$ and $a_{1}$ do not become degenerate in our model.

horizon when the temperature is higher. Hence, this decrease of masses with temperature will be a common feature for all gravitational backgrounds which contain a horizon. However, the precise behavior of the masses as a function of temperature is clearly model dependent. In our case, it depends on parity, the level $n$ and the spin of the meson, as well as on the constituent quark masses. It is interesting to note that generically, for fixed-spin, level and parity, the meson mass runs slower for larger constituent quark masses than for lighter quarks. This behavior is again in qualitative agreement with lattice simulations, see for example [23-26] for quenched results and [27] for a confirmation in unquenched QCD. There, it is found that deeply bound heavy-quark states, such as the $\eta_{c}(J=0)$ or the $J / \Psi(J=1)$, survive up to temperatures which are $\leq 2 T_{\text {crit }}$, where $T_{\text {crit }}$ is the temperature where the light mesons $\rho, \omega$ and $\phi$ melt. For our system, this behavior is a natural consequence of the fact that the constituent quark mass is, roughly speaking, related to the distance of the tip of the probe brane to the horizon. If this distance is increased (while keeping the horizon temperature fixed in units of the radius of the circle $x^{4}$ ), a meson of the same spin, parity and level will correspond to an excitation of the brane which is further away from the horizon, and hence less affected by the temperature. Note that because the phenomenological model of [21] lacks probe branes, it does not have this parameter at its disposal. Hence, it thus seems harder to reproduce this feature of the mesonic spectrum in such ad-hoc models.

One can also follow the mass trajectories for the higher excited modes. This results in Fig. 5. This plot shows interesting qualitative similarities with results in realworld QCD, see e.g. [9], although the latter results are

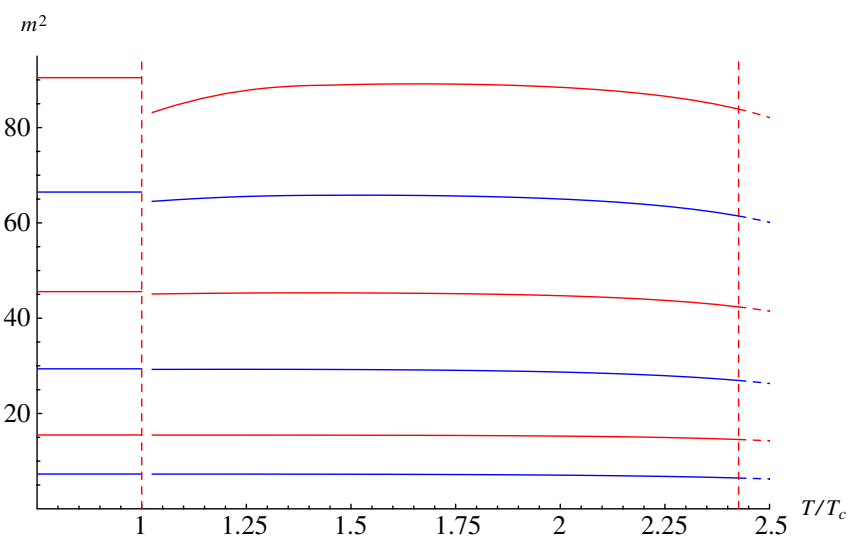

FIG. 5 (color online). Behavior of six lowest-mass vector mesons (for fixed $L / R$ ) as a function of temperature in the intermediate-temperature regime, and a comparison with the masses in the low-temperature phase. Note the jump in the spectrum at the confinement/deconfinement transition, and also note that the masses of the higher excited modes do not exhibit monotonic behavior as a function of temperature.

not in the large- $N_{c}$ limit so one should be careful with a direct comparison. It would be very interesting to extract more quantitative dependence of the meson masses on the aforementioned set of parameters from our model. We leave this question for future investigation.

\section{Phase transition from low to intermediate temperature}

As mentioned before, the mesonic spectrum in the lowtemperature phase is unchanged as the temperature is increased. Although this may sound counterintuitive, it seems to be a generic feature of large- $N_{c}$ theories $[18,19]$. Namely, it was shown in $[18,19]$ that, for example, the value of the chiral condensate $\langle\bar{\psi} \psi\rangle$ is independent of the temperature, essentially because in the confining phase the theory behaves effectively as a gas of noninteracting glueballs and mesons. This behavior is indeed what is reproduced from a generic supergravity solution, since the metric for finite, low temperatures is the same as the metric for zero temperature (note that the same remains true even if $N_{f} \sim N_{c}$ and one constructs the back-reacted geometry). To incorporate finite-temperature effects on the meson spectrum and the value of the chiral condensate, one would need to go beyond the supergravity approximation and deal with genuine finite-temperature string theory.

Let us nevertheless see how the intermediatetemperature and low-temperature phases are connected in our model. To do this, we send the temperature to the critical value $T_{c}$ where the gluonic degrees of freedom get deconfined (i.e. $T \rightarrow 1 / 2 \pi R$ ). Figure 5 shows that the mesonic spectrum at intermediate temperature is not connected to the spectrum in the low-temperature regime. However, since as argued in [3] the transition is first-order, such a jump should be expected. Do notice, however, that the mesons are only mildly affected by the "violent" 
transition of the gluonic degrees of freedom. The mesonic spectrum, though discontinuous, is not rearranged: for every meson state before the transition, there is a corresponding state after the transition. In a certain sense the mesons are merely spectators in this phase transition.

\section{HIGH-SPIN MESONS AT INTERMEDIATE TEMPERATURE}

In this section we examine the properties of high-spin mesons as a function of the temperature. For low-spin mesons, we have seen that the dependence of the string tension on the temperature determines the behavior of the masses. We expect this mechanism to apply to high-spin mesons as well. Therefore we expect that for a meson of fixed spin and low excitation number, the mass will decrease as a function of temperature. We will indeed find this behavior. However, there is now an additional interesting phenomenon, which only appears for high-spin mesons, since their spin can vary significantly. Spin is a parameter which controls the length of the string as well the distance of the tip of the string to the horizon. Hence, a natural question arises as to whether the confinementdeconfinement transition of mesons (i.e. the process of "dissociation" of the mesons), takes place at the same temperature for all mesons, irrespective of their masses and spins? Intuitively, one expects that higher-spin mesons will dissociate first, given that they "hang" closer to the horizon. The question is then what is the critical value of the spin as a function of temperature?

To answer the questions posed in the previous paragraph, let us consider large spinning mesons at finite temperature. These are modeled by rotating $U$-shape strings hanging from the probe brane in the middle picture of Fig. 1. The relevant part of the background metric (4) is

$$
\begin{aligned}
\mathrm{d} s^{2}= & \left(\frac{u}{R_{\mathrm{D} 4}}\right)^{3 / 2}\left(-f(u) \mathrm{d} t^{2}+\mathrm{d} \rho^{2}+\rho^{2} \mathrm{~d} \varphi^{2}\right) \\
& +\left(\frac{R_{\mathrm{D} 4}}{u}\right)^{3 / 2} \frac{\mathrm{d} u^{2}}{f(u)} .
\end{aligned}
$$

We go to the static gauge for the string action and make the following ansatz for the rotating configuration, ${ }^{4}$

$$
t=\tau, \quad \rho=\sigma, \quad u=u(\rho), \quad \varphi=\omega \tau .
$$

We see that the ansatz has the the same form as in the zerotemperature case [28]. Hence, just like for the Wilson loop, the only effect of finite temperature will be that the details of the shape $u(\sigma)$ change as the temperature is increased.

One could wonder why such an unmodified ansatz is correct, given that the string is moving with angular ve-

\footnotetext{
${ }^{4}$ When solving the equations of motion numerically, the fact that this gauge leads to a $\mathrm{d} u / \mathrm{d} \sigma$-derivative which blows up at the flavour brane is rather inconvenient. For numerical purposes, a better gauge choice is, for instance, given by $\rho+u=\sigma$.
}

locity, and velocity dependence has been shown to modify the zero-temperature ansatz for vertical strings due to "dragging" effects [11-17]. To answer this question we need to see if the ansatz (22) allows for physical solutions. If it does, this would imply that angular velocity of a meson in a hot medium does not lead to the distortion of rotation, i.e. one does not need to apply a force to maintain the rigid rotation: an initially rigidly rotating meson continues to rotate rigidly. This of course does not mean that the $u(\rho)$ profile is unaffected by the temperature. A separate question is what is the effect of linear motion of this color singlet. This will be explored in section IV B.

To answer the first question, about the possibility of having a rigidly rotating meson in a quark-gluon plasma, let us compute the action for the meson (22). This ansatz leads to

$$
S=\int \mathrm{d} \tau \mathrm{d} \rho \sqrt{\left(\frac{u}{R_{\mathrm{D} 4}}\right)^{3}\left(\rho^{\prime 2}+\frac{u^{12}}{f(u)} \frac{R_{\mathrm{D} 4}^{3}}{u^{3}}\right)\left(f(u)-\rho^{2} \omega^{2}\right) .}
$$

Before we analyze the resulting equations of motion, let us first make a few general remarks. We see that positivity of the argument of the square root in (23) requires that $f(u)>$ $\rho^{2} \omega^{2}$. This in turn means that for a given angular frequency $\omega$, the string solution $u(\rho)$ has to lie above the curve

$$
u(\rho) \geq \frac{u_{T}}{\left(1-\rho^{2} \omega^{2}\right)^{1 / 3}} .
$$

In Fig. 6 we have depicted these curves for various values of $\omega$. Firstly, we see that the tip of any string (the $\rho=0$ point) is in principle allowed to touch the horizon for any angular frequency $\omega$. Secondly, for given $\omega$, the maximal allowed extent of the string is determined by the intersection of the curve with $u_{0}$, and is given by

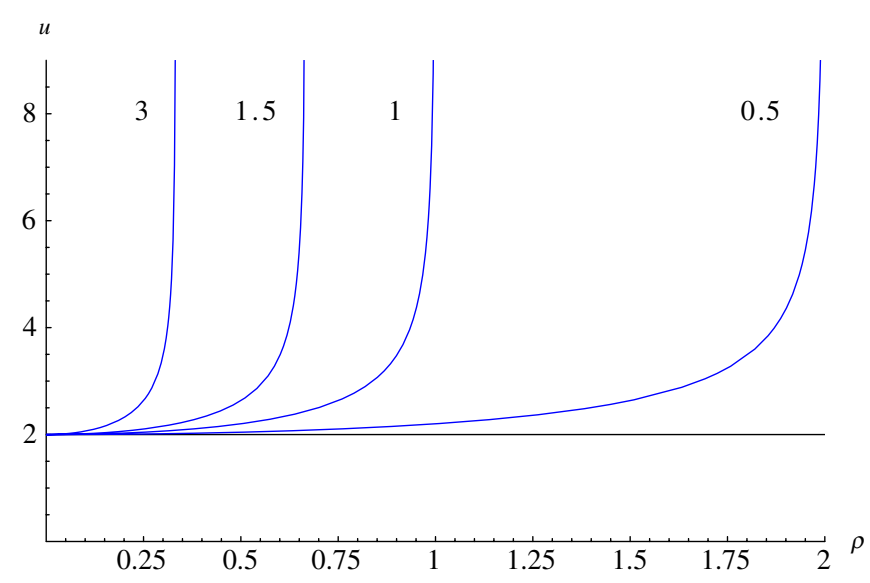

FIG. 6 (color online). The boundary curve (24). Rotating strings have to lie above this curve in order for their action to be real. The various curves correspond to various values of the frequency $\omega$. The horizon is located at $u_{T}=2$. These curves are independent of the probe-brane location. 

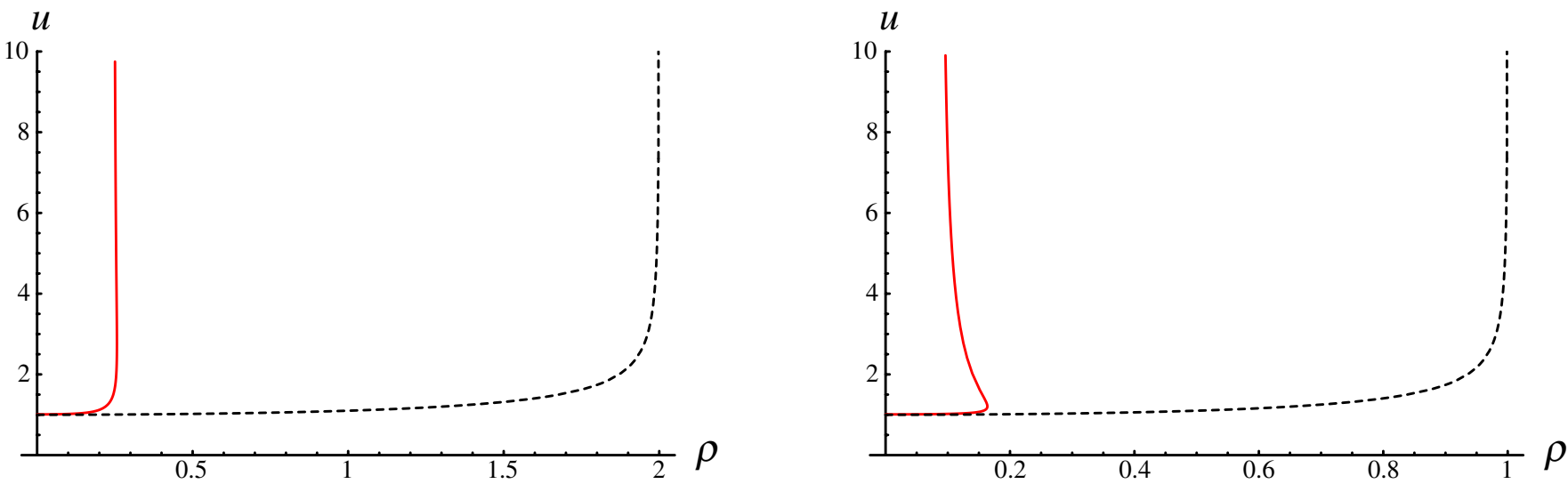

FIG. 7 (color online). Comparison of numerical solutions (solid curves) with the boundary curve (24) (dashes), for $\omega=0.5$ and $\omega=1$ respectively. Although the numerical solution lies manifestly above the boundary curve, as it should, it deviates quite a bit from it. The numerical solutions where obtained by fixing $u(0)=u_{T}+\epsilon$ for a small $\epsilon$, as well as $u^{\prime}(0)=0$.

$$
\rho_{\max }=\frac{1}{\omega} \sqrt{1-\frac{u_{T}}{u_{0}}}
$$

(One could have hoped that the boundary curve (24) becomes a good approximation to the real string solution in some regime. However, this does not seem to be the case, as is easily seen from a comparison of this curve with some real solutions, Fig. 7.)

The equations of motion following from the action (23) are given by

$$
\begin{aligned}
-2 \sqrt{\cdots} \frac{\mathrm{d}}{\mathrm{d} \sigma}\left(\frac{1}{\sqrt{\cdots}}\right. & \left.\frac{u^{\prime}}{f(u)}\left(f(u)-\rho^{2} \omega^{2}\right)\right) \\
+f^{\prime}(u)\left(\frac{u^{\prime 2} \rho^{2} \omega^{2}}{f(u)^{2}}\right. & \left.+\frac{u^{3}}{R_{\mathrm{D} 4}^{3}}\left(\rho^{\prime}\right)^{2}\right) \\
& +\frac{3 u^{2}}{R_{\mathrm{D} 4}^{3}}\left(\left(\rho^{\prime}\right)^{2}-\left(\rho^{\prime}\right)^{2} \rho^{2} \omega^{2}\right)=0,
\end{aligned}
$$

where $\sqrt{\cdots}$ is the density of Nambu-Goto action (23). One can verify that varying the action first and then inserting the ansatz yields the same result. In addition we have to take care of the boundary terms. If one were not yet in the static gauge, one would impose Neumann boundary condition for the $\rho$ direction and Dirichlet in the $u$-direction. These two, when combined imply that string end "orthogonally" on the brane world volume $\mathrm{d} u / \mathrm{d} \rho \rightarrow \infty$.

Finally, the expressions for the energy and the angular momentum carried by the string are given by

$$
\begin{gathered}
E=\int \mathrm{d} \sigma \frac{1}{\sqrt{\cdots}}\left(\left(\frac{u}{R_{\mathrm{D} 4}}\right)^{3} f(u)\left(\rho^{\prime}\right)^{2}+u^{\prime 2}\right), \\
J=\int \mathrm{d} \sigma \frac{1}{\sqrt{\cdots}} \omega \rho^{2}\left(\left(\frac{u}{R_{\mathrm{D} 4}}\right)^{3} \rho^{\prime 2}+\frac{u^{\prime 2}}{f(u)}\right),
\end{gathered}
$$

where $\sqrt{\cdots}$ is once more the density of the Nambu-Goto action (23).

\section{A. Properties of the spectrum and melting of large-spin meson}

Because of the complexity of Eq. (26), we will analyze the spectrum of large spinning strings numerically. Nevertheless, interesting qualitative features can easily be extracted from this analysis.

Let us first discuss the spectrum for fixed temperature. By analysing the shapes of the string for various values of $\omega$, we see that as $\omega$ decreases, i.e. the spin of the meson increases, the string endpoints get more and more separated, and the $U$-shaped string penetrates deeper to the horizon. It becomes more and more rectangular shaped. A few characteristic shapes, for mesons with fixed $T$ and $m_{q}$ but varying $\omega$ (or equivalently $J$ ) are depicted in Fig. 8.

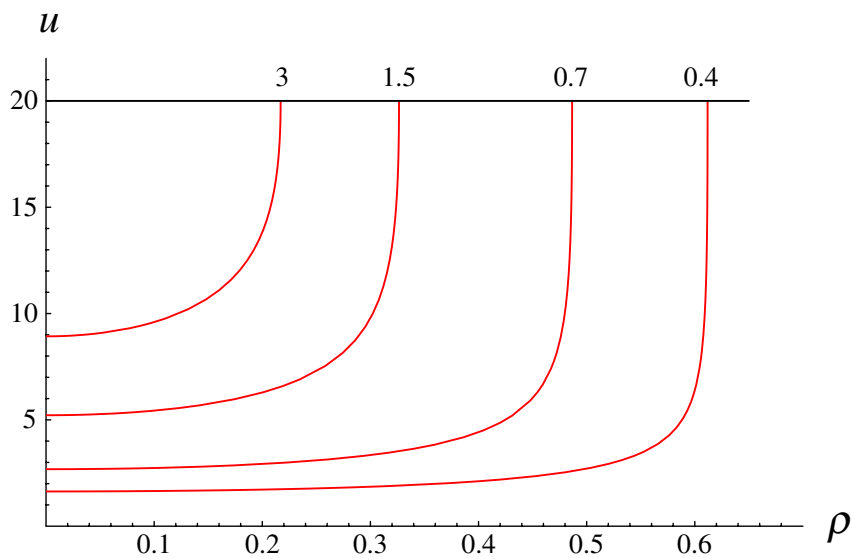

FIG. 8 (color online). Shapes of rotating strings with fixed position of the flavour brane $u_{0}=20$ (i.e. almost fixed $L / R$, see Fig. 2) and fixed temperature $u_{T}=1$, for various values of the frequency $\omega$. 

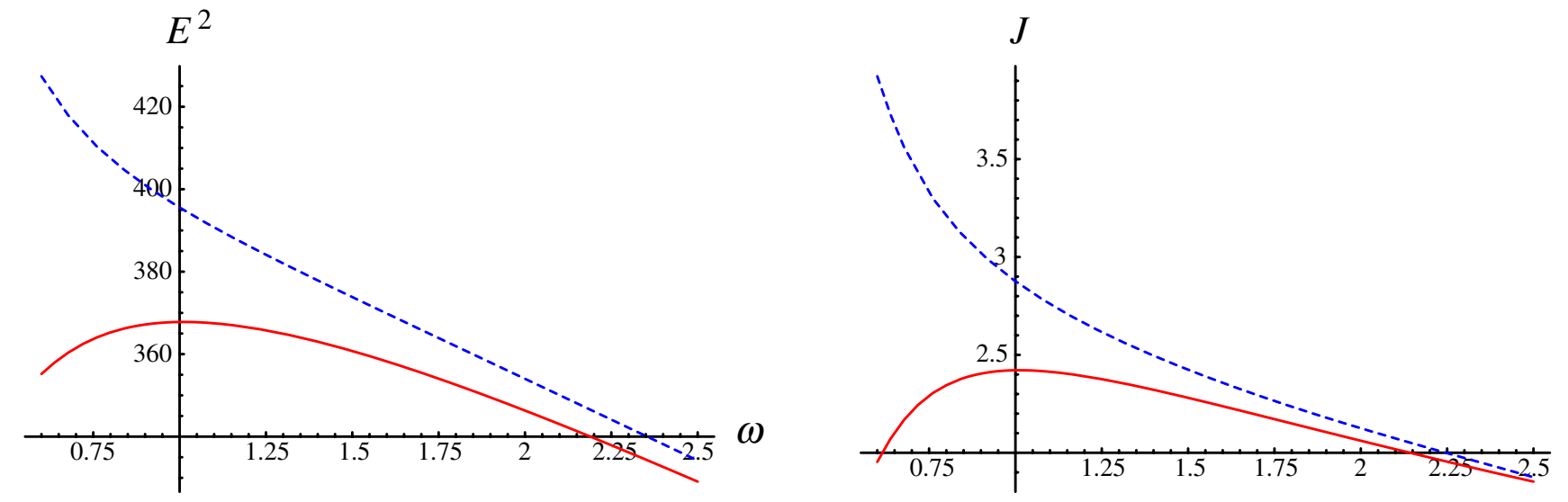

FIG. 9 (color online). The energy (squared) and angular momentum of $U$-shaped strings, as a function of the frequency $\omega$. The dashed (blue) curves are for zero temperature, while the solid (red) curves are for the intermediate-temperature regime.

Integration of Eq. (26) yields the dispersion relation in parametric form, i.e $E(\omega)$ and $J(\omega)$ at fixed temperature. These curves, together with similar curves at zero temperature, are shown in the plots in Fig. 9. We see that at zero temperature, the spin increases as the angular frequency $\omega$ is decreased (just like in flat space, where this behavior is essentially determined by the condition $\omega L=1$ which says that the endpoints move with the velocity of light). As the spin increases, the string hangs deeper into the geometry and hence the energy of the meson goes up. The same happens at non-zero temperature for large values of $\omega$, i.e. when the string is short and thus far away from the horizon. However, as $\omega$ is decreased, even though the length of the string continues to grow, the effective tension of the parts of string in the region near the horizon decreases rapidly. This effects leads to a slower growth of spin and energy, and hence to the appearance of a maximum in the energy and spin. This is shown in the solid (red) curves of the plots in Fig. 9.

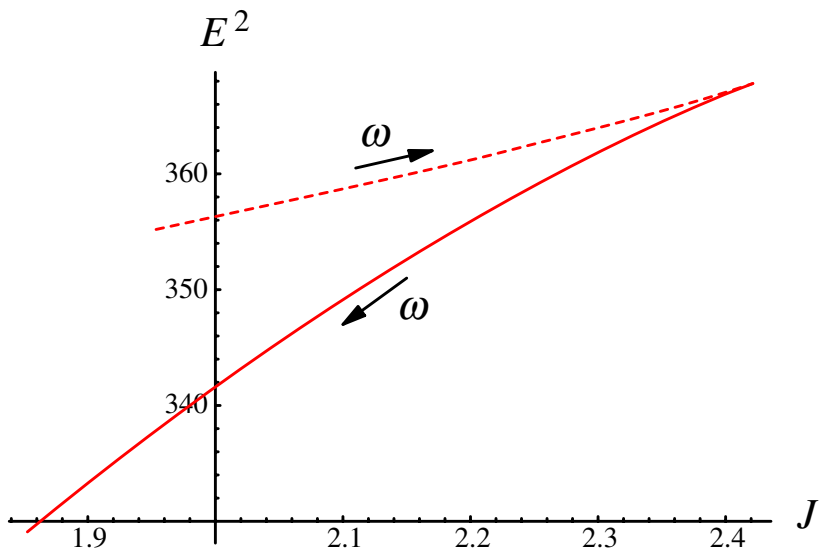

When we eliminate the variable $\omega$ from the two plots in Fig. 9, to obtain a plot of the energy versus the spin, we get Fig. 10. The appearance of a maximum in energy and spin is again clearly visible. We also see that the two states with identical spin $J$ actually have different energy: the ones with smaller $\omega$ are more energetic than the ones with larger $\omega$. Therefore, the upper branch to the left of the maxima in the plots of Fig. 9 is presumably unstable and will decay to the lower branch.

Apart from the two configurations in Fig. 10, with $E_{1}$ and $E_{2}$ for fixed $J$, there is yet another configuration one may consider; this configuration is in spirit closer to the dissociation of Wilson loops. Namely, one could consider the configuration of two dragged single quarks (to be described in more detail in the next section), with linear velocities $v$ which are such that the whole system carries the same angular momentum as our mesons. Then, one could compare the energy of such a configuration with the energy of the meson before dissociation. However, as

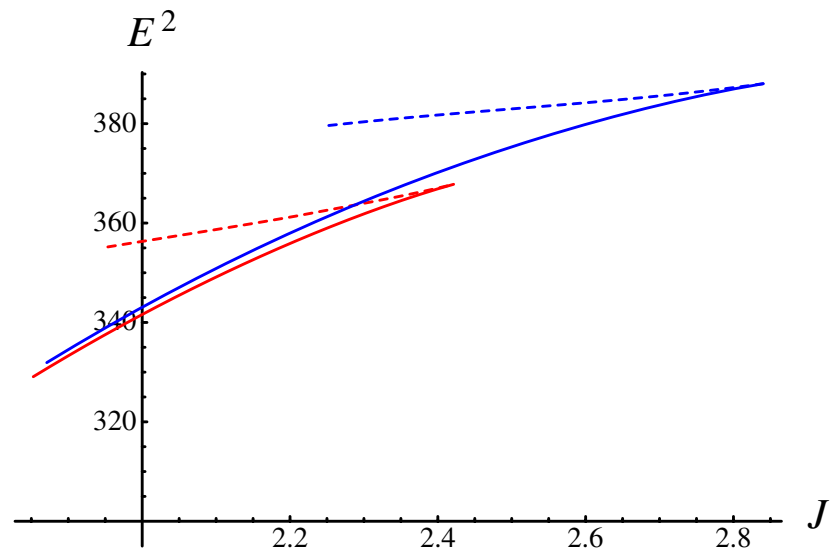

FIG. 10 (color online). Left panel: Energy versus angular momentum for $L / R=0.24$ in the intermediate-temperature phase, $T / T_{c}=\sqrt{2}$. The lower segment (solid) is for $\omega>1$, the upper one (dashed) for $\omega<1$. There is a maximum reachable spin. Right panel: Similar plot, but now for two different temperatures. The blue (upper) curves are for a lower temperature $T / T_{c}=1$, the red (lower) curves for a higher temperature $T / T_{c}=\sqrt{2}$. Observe that for a fixed spin, the energies go down as the temperature goes up. 


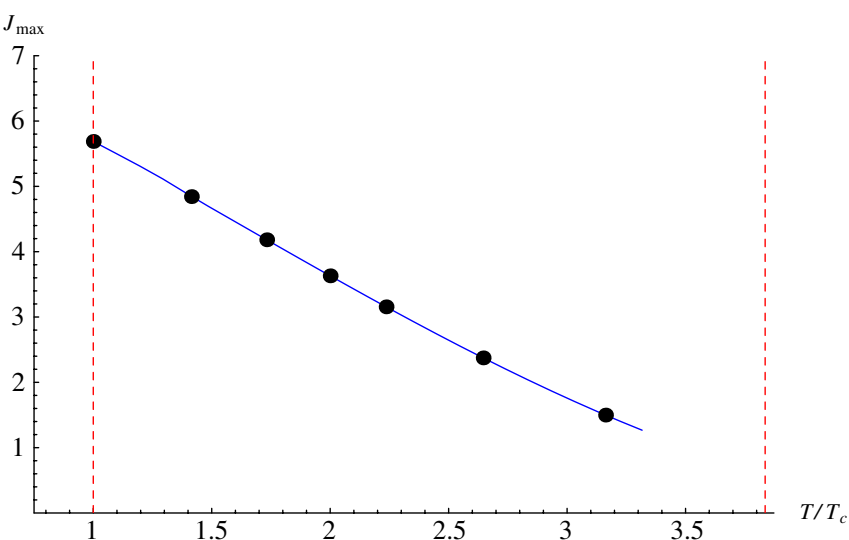

FIG. 11 (color online). Analysis of maximum spin for $U$-shaped strings in the intermediate-temperature regime, as a function of temperature, for fixed $L / R=0.24$. Angular momentum is normalized as in (28). There is of course a divergence as $T / T_{c} \rightarrow 0$, but before that point is reached, one encounters the phase transition to the low-temperature background (vertical dotted line at $T / T_{c}=1$ ).

shown in [11] this configuration always carries infinite energy, and thus cannot be the one which acts as initial data for the dissociated meson. ${ }^{5}$

The plot of $J$ versus $\omega=\omega\left(u_{\text {bottom }}\right)$ is similar to the plot of the interquark distance versus the minimum distance to the horizon found for Wilson loops. For Wilson loops, it was found that there is a maximum interquark distance. For spinning strings, such a maximum distance appears too. However, this maximum distance is not reached for the same value of $\omega$ as the value for which the maximum spin is attained. A priori, this is a source of worry, since it could mean that the relation between $J$ and the interquark distance $L$ is not monotonic. However, we have verified numerically that the maximum interquark distance always occurs in the unphysical part of the spectrum; the relation between $J$ and $L$ is monotonic in the physical regime (this is somewhat similar to the monotonicity of $L$ versus $y_{T}$ depicted in Fig. 2).

From the analysis above, we see that for a fixed temperature there is a maximal value of the spin which a meson can carry. It is natural to interpret the temperature at which this happens as the critical temperature at which a meson of spin $J_{\max }$ melts. Thus we conclude that (as intuitively expected) the dissociation temperature of large-spin mesons is spin dependent. As the temperature increases, the maximal value of the spin that a meson can carry decreases (see Fig. 11), i.e. for given quark mass, higher-spin mesons melt at lower temperature. We also see that for meson of fixed angular momentum, the energy decreases as a function of temperature, see Fig. 12. This is the same behavior as was observed for the low-spin mesons. However, the

\footnotetext{
${ }^{5}$ In this sense the dissociation of spinning strings is different from the dissociation of Wilson loops $[29,30]$.
}

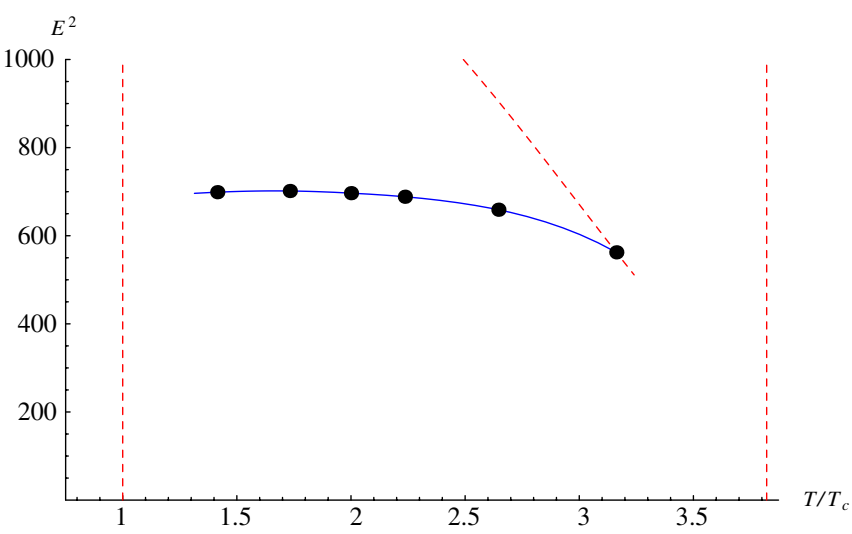

FIG. 12 (color online). Analysis of the temperature dependence of the energy of $U$-shaped strings. Displayed are the squared energies of a state of $\operatorname{spin} J \approx 1.5$, which dissociates at $T / T_{c}=3.16$ when $L / R=0.24$. The diagonal dotted curve shows the maximum possible energy for a given temperature. Note the qualitative similarity with the low-spin plot, Fig. 5.

four-dimensional size of the meson (i.e. the distance between the two endpoints of the $U$-shaped string measured along the probe-brane) increases as the temperature goes up; see Fig. 13. Finally, by considering the shapes of strings at different temperatures (Fig. 14) we observe that, as the temperature is increased, the largest allowed strings become less and less rectangular shaped, and more and more $U$-shaped, thus leading to the breakdown of the fourdimensional picture of a string with two massive, pointlike strings attached to it [28]. This is an important observation if one wants to construct four-dimensional effective string models for mesons at finite temperature.

\section{B. Drag effects for mesons}

It was recently observed that the drag force experienced by free quarks moving through a hot gluon plasma can be

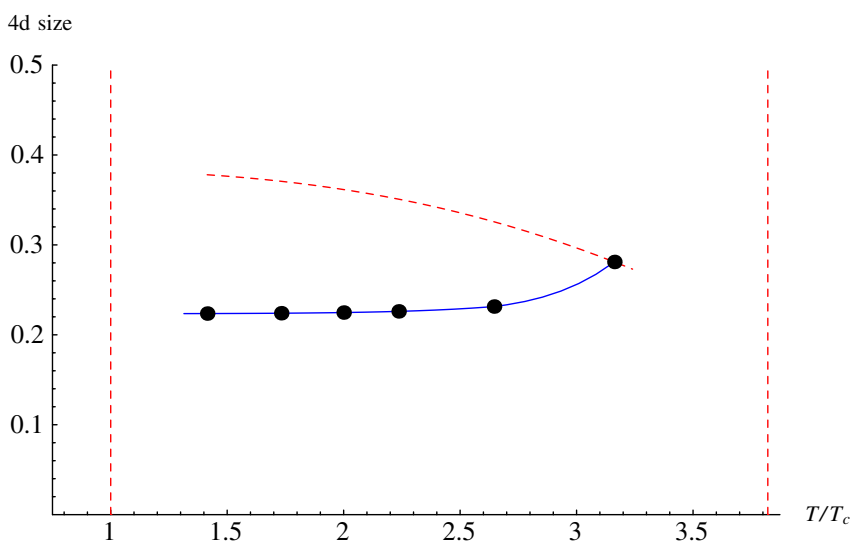

FIG. 13 (color online). The four-dimensional size of a largespin meson of fixed spin as a function of $T / T_{c}$ : when the temperature goes up, the meson expands. The dotted curve denotes the size of the maximum-spin meson at a given temperature. 

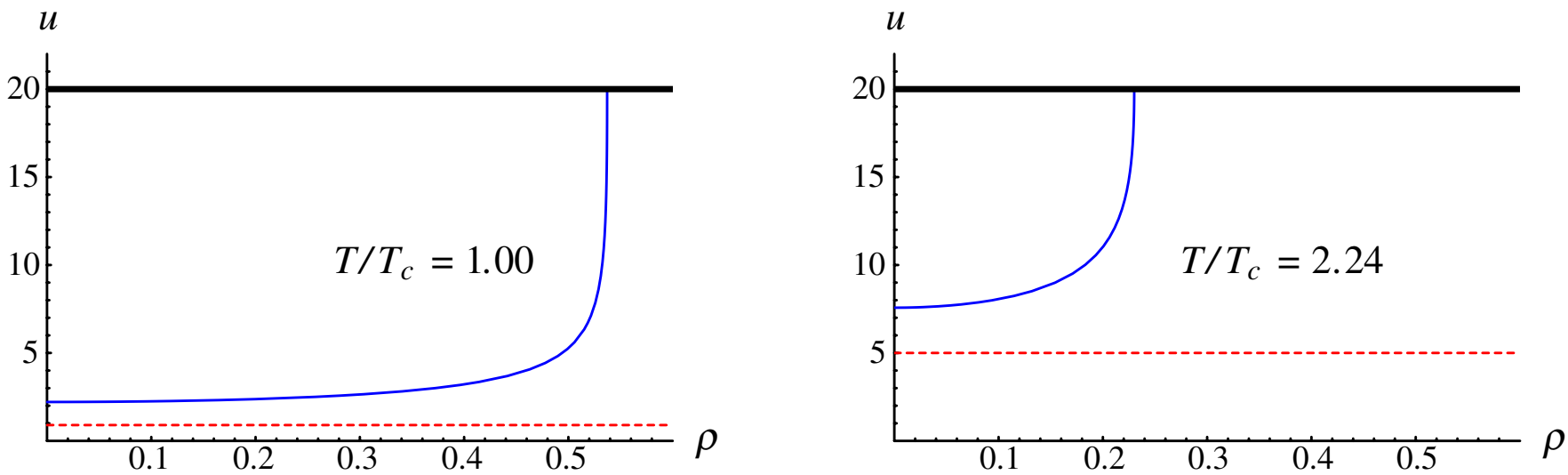

FIG. 14 (color online). The two plots illustrate the shape of the maximum- $J$ strings at two selected temperatures (dotted horizontal red lines denote the horizon, solid black lines the flavour brane).

modeled in string theory [11-17]. In this setup, the quark is a single string ending with one end on the flavour brane and extending all the way down to the horizon. Because a strictly vertical string moving rigidly through the background would not have a real action (23), the string has to be bent when it is "pushed" through the plasma. In addition the bent string does not end anymore orthogonally on the brane. This means that one has to apply a force on the string endpoint, or in other words, one has to "drag" the string in order to keep it moving.

In more detail, this conclusion follows from considering the string action evaluated on the "naive" ansatz describing a rigid straight string moving with velocity $v_{x}$,

$$
t=\tau, \quad u=\sigma, \quad x=v_{x} t .
$$

Plugging this ansatz into the action (23), we get

$$
S_{\text {quark }}=\sqrt{1-\frac{v_{x}^{2}}{f(u)}}
$$

For this expression to be real, a purely vertical string cannot stretch lower than

$$
u>u_{c}, \quad u_{c}=\frac{u_{T}}{\left(1-v_{x}^{2}\right)^{1 / 3}},
$$

and it clearly cannot stop there when there is no second flavour brane present. The true shape is obtained by adding a $\sigma$-dependent term to the ansatz for $x$. The string now bends as it reaches down to the horizon. The bent, moving string does not, however, satisfy Neumann nor Dirichlet boundary conditions, neither at the horizon nor at the probe brane. Hence the world-sheet momentum does not vanish at the string endpoints: there is a world-sheet momentum flow into the horizon, which has to be "added" by a force dragging the string on the upper, probe-brane side. This effect translates to a drag force in the gauge theory picture.
Hence in summary, we see that there are two effects happening as one tries to move a single string in the hot background: firstly, the string shape is modified in a way which depends on the temperature and velocity, and secondly, in order to preserve the motion one needs to apply a force.

It is a natural question to ask if such effects are also exerted on spinning mesons, as the temperature is turned on. One might expect that even a simple rotating motion experiences a drag effect, but the analysis of the previous subsection shows that this effect does not exist. Instead, the rotating string is always sufficiently high above the curve beyond which the action would turn imaginary. No worldsheet momentum gets lost behind the horizon. On the other hand, the first effect is still present. The bending of the rotating meson does depend both on the angular velocity and on the temperature.

However, there is another type of motion which one can consider, and which is more similar to the moving-quark situation. Namely, one can consider a linear motion of the meson in a direction orthogonal to the plane of rotation. ${ }^{6} \mathrm{~A}$ suitable ansatz for this motion is

$$
\begin{gathered}
t=\tau, \quad \rho=\sigma, \quad u=u(\rho), \\
\varphi=\omega \tau, \quad y=v_{y} \tau .
\end{gathered}
$$

In this case the action becomes

$$
S=\int \mathrm{d} \tau \mathrm{d} \rho \sqrt{\frac{u^{3}}{R_{\mathrm{D} 4}^{3}}\left(1+\frac{u^{\prime 2}}{f(u)} \frac{R_{\mathrm{D} 4}^{3}}{u^{3}}\right)\left(f(u)-\rho^{2} \omega^{2}-v^{2}\right) .}
$$

\footnotetext{
${ }^{6}$ One could also consider motion in the direction in which the string rotates. In this case the equations of motion are much more involved. We do, however, expect that the conclusion reached by studying this motion will be similar to those reached by studying motion in a transverse direction.
} 

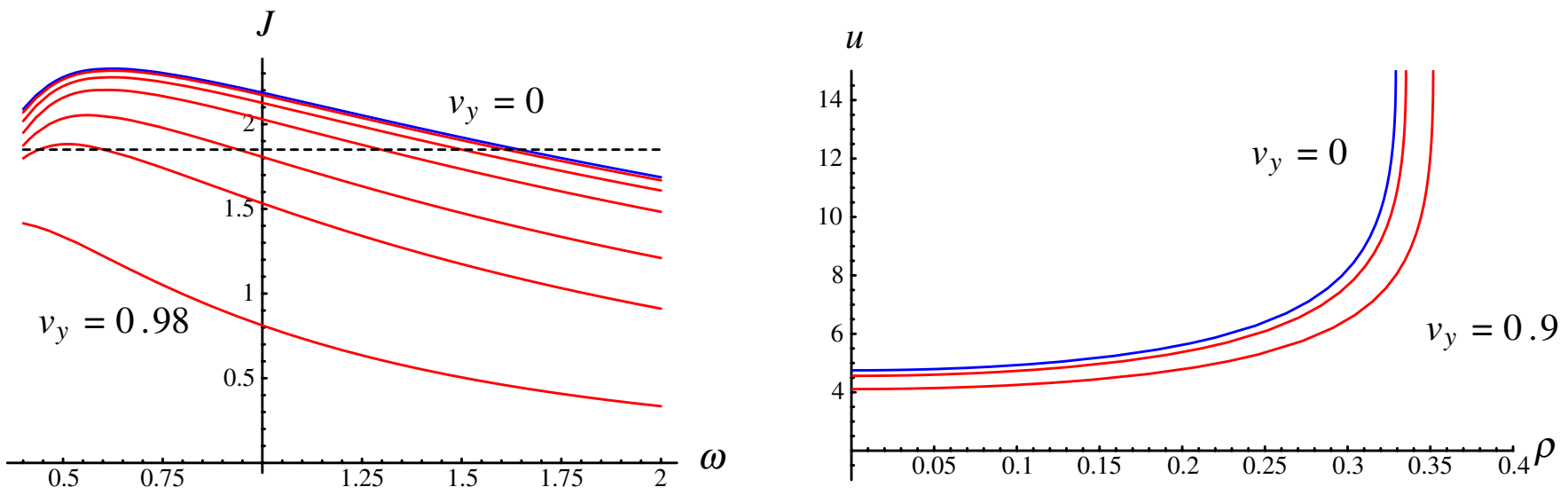

FIG. 15 (color online). Analysis of the effect of a transverse velocity on spinning $U$-shaped strings. The left panel depicts the relation between $J$ and $\omega$ for fixed position of the flavour brane. The upper curve is for $v_{y}=0$, while the other curves display results for increasing values $v_{y}=0.2,0.4,0.6,0.8,0.9,0.98$. The panel on the right shows the effect of a transverse velocity on the shape, keeping the quark masses and spin fixed (corresponding to the dashed black line in the left panel).

The only modification with respect to the rotating meson is the addition of a term " $-v^{2}$ " to the last factor under the square root. The condition for the action to be real is now

$$
u \geq \frac{u_{T}}{\left(1-\rho^{2} \omega^{2}-v^{2}\right)^{1 / 3}} .
$$

Hence, if the ansatz (32) makes sense, there should exist a solution to the equations of motion such that the spinning configuration lies entirely above this curve. If that is not the case, the ansatz has to be modified.

We have verified that the equations of motion obtained from (33) are the same as those of the full system after insertion of the ansatz (32). Subsequently, we have integrated the equations of motion using the same techniques as for the zero-velocity case. The results are depicted in Fig. 15. The left panel shows that, as the transverse speed is increased, the maximum possible spin decreases. If we take a state of fixed spin $J$ and look at how its shape changes as we increase the transverse speed, we obtain the second panel in Fig. 15 (this corresponds to following the states which lie on the black dotted curve in the first panel). All these configurations, up to the maximum-spin ones, lie safely above the limiting curve (34). Note how the size of a fixed-spin meson increases slightly as the transverse velocity is turned on.

Thus, the important physical conclusion seems to be that color singlet states do not experience any drag effect. From the field-theoretic point of view this makes sense, as the absence of a color monopole moment means that these states do not couple directly to gluon degrees of freedom. Because of the absence of drag, mesons do not experience any energy loss when propagating through the quark-gluon plasma: no force is necessary to keep them moving with fixed velocity. ${ }^{7}$ Note moreover that the moving meson does not need to bend in the direction of motion, i.e. there is no $y(u)$ term present in (32). However, the shape of the meson in the $(\rho, u)$ plane certainly is velocity dependent. As shown on the right panel of Fig. 15, the distance to the horizon decreases, leading to a lower melting temperature.

Figure 15 (left panel) also shows that the maximum spin goes down as the velocity goes up. There is thus a critical velocity beyond which a meson of fixed spin has to dissociate. Similarly, the four-dimensional size of the meson decreases with increasing velocity, as can be seen in Fig. 16. The data is approximated rather well by the relation

$$
L_{\max -\operatorname{spin}}^{4 d}(v) \approx L_{\max -\operatorname{spin}}^{4 d}(v=0) \cdot\left(1-v^{2}\right)^{1 / 4} .
$$

This fit was motivated by the analytic results of [31], in which a similar dependence on the velocity was found for the screening length, or more precisely, the maximum interquark distance for Wilson loops in an AdS black hole background. See also related results in [32].

Finally, one should keep in mind that our analysis in this section is purely classical, and restricted to the sigma model, without any coupling to the fields on brane. In principle there will be classical (and quantum) energy loss due to radiation effects on brane, because the meson is essentially a dipole. However, the interpretation of this

\footnotetext{
${ }^{7} \mathrm{We}$ should emphasize that what we have computed here is different from the computation of [17]. In that paper, a situation is considered in which two quarks, being endpoints of a "twisted" Wilson loop, move away from each other. The required energy influx is higher at finite temperature, giving rise to a four-dimensional drag force. For us, the two quarks which make up the meson move in the same direction, and we do not change the $U$-shaped string as a function of time.
} 


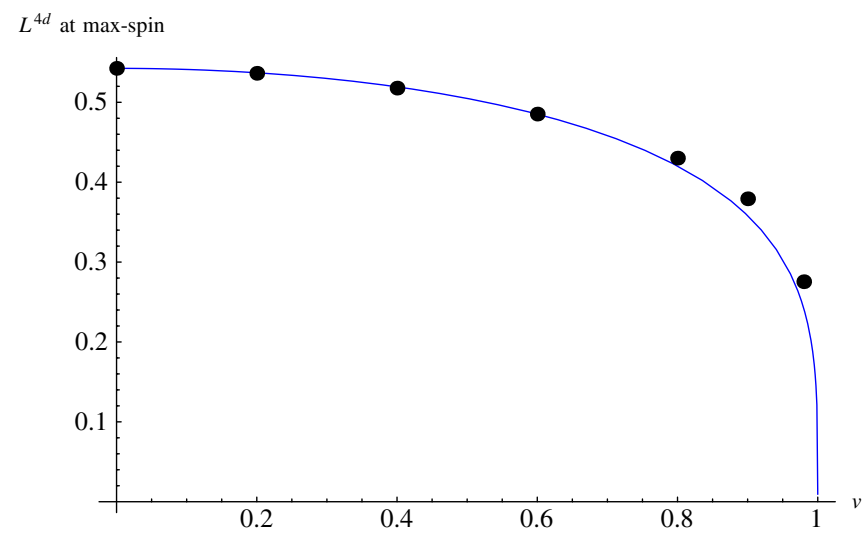

FIG. 16 (color online). The behavior of the four-dimensional size $L^{4 d}$ for maximum-spin mesons as a function of the transverse velocity (for a particular choice of $m_{q}$ and $T=T_{c}$ ). The blue curve depicts $L^{4 d}(v=0) \cdot\left(1-v^{2}\right)^{1 / 4}$, as obtained analytically for Wilson loops in [31].

energy loss is different, as it corresponds to the decay of high-spin mesons into low-spin ones [33-35], both at zero and at non-zero temperature.

\section{SPECTRUM AT HIGH TEMPERATURE}

Finally, let us briefly discuss the high-temperature phase. In this phase, the minimal free energy of the system is attained when the two stacks of branes are disconnected (see the third panel of Fig. 1). The profile of the left and right stacks of branes is characterized by $u^{\prime} \equiv \mathrm{d} u / \mathrm{d} x^{4} \rightarrow$ $\infty$ and the induced metric on the probe branes and probe antibranes takes the form

$$
\begin{aligned}
d \hat{s}_{\text {high }}^{2}= & \left(\frac{u}{R_{\mathrm{D} 4}}\right)^{3 / 2}\left[-f(u) \mathrm{d} t^{2}+\delta_{i j} \mathrm{~d} x^{i} \mathrm{~d} x^{j}\right] \\
& +\left(\frac{R_{\mathrm{D} 4}}{u}\right)^{3 / 2} \frac{1}{f(u)} d u^{2}+\left(\frac{R_{\mathrm{D} 4}}{u}\right)^{3 / 2} u^{2} \mathrm{~d} \Omega_{4}^{2},
\end{aligned}
$$

i.e. it is the same as the background metric without the $g_{44}\left(\mathrm{~d} x^{4}\right)^{2}$ term. Considering now the action of the gauge fields on each of the two stacks of branes, it is straightforward to see that the action will be similar to the one at intermediate temperature, but with $\gamma=1$. The differential equation for the modes is now

$$
-u^{1 / 2} f(u)^{1 / 2} \partial_{u}\left(u^{5 / 2} f(u)^{1 / 2} \partial_{u} \psi_{(n)}\right)=R_{\mathrm{D} 4}^{3} m_{n}^{2} \psi_{(n)} .
$$

which differs from the one at intermediate temperature (14) by the absence of the $\gamma$ factors. The normalization conditions are similarly stripped off their $\gamma$ factors,

$$
\begin{aligned}
& \int_{u_{T}}^{\infty} \mathrm{d} u u^{4} f^{-1 / 2}(u)\left(\frac{u}{R_{\mathrm{D} 4}}\right)^{9 / 2} \psi_{(m)} \psi_{(n)}=\delta_{m n}, \\
& \int_{u_{T}}^{\infty} \mathrm{d} u u^{4} f^{-1 / 2}(u)\left(\frac{R_{\mathrm{D} 4}}{u}\right)^{3 / 2} \phi^{(0)} \phi^{(0)}=1 .
\end{aligned}
$$

The first thing to observe now is that the massless pion disappears from the spectrum. This mode, which would be given by $\phi^{(0)}=u^{-5 / 2} f(u)^{-1 / 2}$, is no longer normalizable. Computation of its norm leads to the integral

$$
\int_{u_{T}}^{\infty} \mathrm{d} u u^{5 / 2} f(u)^{-1 / 2}\left|u^{-5 / 2} f(u)^{-1 / 2}\right|^{2},
$$

which, while convergent at the upper boundary, is divergent at the lower boundary because $f(u) \sim \sqrt{u-\bar{u}_{T}}$ for $u \sim u_{T}$. Here, it is crucial that the D8-branes extend all the way down to the horizon at $u=u_{T}$ (recall that at finite temperature, these $f$-factors did not lead to a divergence because the integral had a lower limit at $u=u_{0}$ ). In accordance with the fact that chiral symmetry is restored in the high-temperature phase, we see that the Goldstone boson has disappeared.

In order to see whether the remainder of the spectrum is discrete or continuous, we write our Eq. (37) as (we set $R_{\mathrm{D} 4}=u_{T}=1$ from now)

$$
f(u)^{1 / 2} \partial_{u}\left(u^{5 / 2} f(u)^{1 / 2} \partial_{u} \psi_{(n)}\right)+u^{-1 / 2} m^{2} \psi_{(n)}=0 .
$$

If we expand this near $u=1$ we get

$$
3 \sqrt{u-1} \partial_{u}\left(\sqrt{u-1} \partial_{u} \psi(u)\right)+m^{2} \psi(u)=0 .
$$

The general solution to this equation is

$$
\psi(u)=c_{1} \cos \left(2 m \sqrt{\frac{u-1}{3}}\right)+c_{2} \sin \left(2 m \sqrt{\frac{u-1}{3}}\right),
$$

which is obviously finite at the horizon for any choice of the constants $c_{1}$ and $c_{2}$. Moreover, these modes are regular in the norm (38).

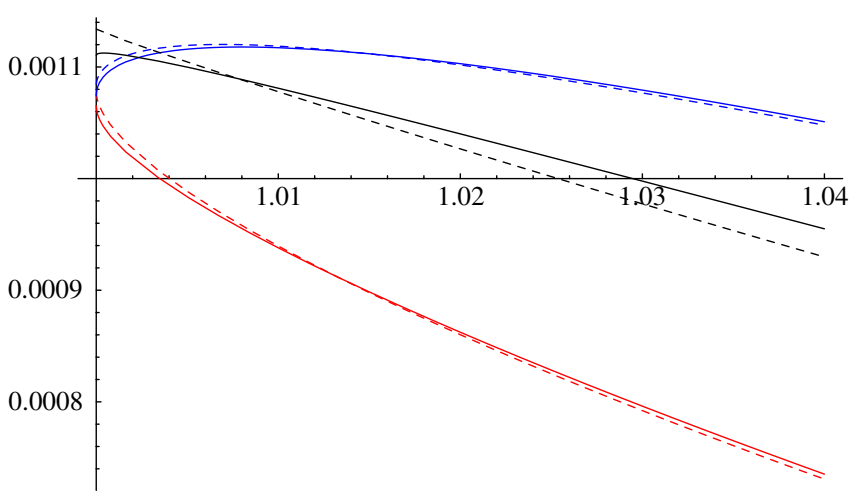

FIG. 17 (color online). High-temperature region: comparison of the exact modes (42) near the horizon (dashed curves) and modes obtained by shooting from infinity (solid curves) for $m^{2}=2.7$ (lower curves), $m^{2}=3.0$ (middle curves) and $m^{2}=$ 3.3 (upper curves). Note that the exact solution always has a nonzero derivative at the origin; the $m^{2}=3.0$ curve obtained from shooting has zero derivative but this is an artifact of the numerical procedure. 
If one now shoots from infinity, starting with the normalizable mode for a fixed $\mathrm{m}^{2}$, one can always match this to a regular solution at the horizon because there are two free parameters available there. In Fig. 17 we compare the numerical solutions with the approximations (42). Because there are two regular solutions at the horizon the spectrum will be continuous in the high-temperature phase.

Note that this type of analysis could in principle be made also for the zero- and intermediate-temperature phases. In those cases, one will find that again both solutions are regular at the tip of the probe brane. However, there is now an additional condition, namely, that the solution can be continued to the other side of the probe brane and remain normalizable. So in these two phases, it is not regularity at the tip, but rather the condition that the function is either odd or even which makes the spectrum discrete.

\section{ACKNOWLEDGMENTS}

We would like to thank Ofer Aharony for many illuminating discussions and comments on a draft of this paper. J. S. would like to thank E. Shuryak for a useful discussion. The work of J.S. was supported in part by the Israel Science Foundation (grant number 03200306) and by a grant of DIP (H.52).

Note added. - After publication of the first version of this paper, [31] appeared, which contains a computation of the screening length at finite temperature (the maximum interquark distance for Wilson loops). Our results leading to Fig. 15 agree with the analytic behavior $L_{\max \text {-spin }}^{4 d}(v) \sim$ $L_{\max \text {-spin }}^{4 d}(v=0)\left(1-v^{2}\right)^{1 / 4}$ found there; we have added Fig. 16 and the paragraph around (35) to make this more explicit.
[1] T. Sakai and S. Sugimoto, Prog. Theor. Phys. 113, 843 (2005).

[2] T. Sakai and S. Sugimoto, Prog. Theor. Phys. 114, 1083 (2006).

[3] O. Aharony, J. Sonnenschein, and S. Yankielowicz, hepth/0604161.

[4] A. Parnachev and D. A. Sahakyan, Phys. Rev. Lett. 97, 111601 (2006).

[5] P. de Forcrand et al. (QCD-TARO), Phys. Rev. D 63, 054501 (2001).

[6] F. Karsch, D. Kharzeev, and H. Satz, Phys. Lett. B 637, 75 (2006).

[7] C.-Y. Wong, hep-ph/0606200.

[8] F. Karsch, Nucl. Phys. B, Proc. Suppl. 83, 14 (2000).

[9] E. Shuryak, Prog. Part. Nucl. Phys. 53, 273 (2004).

[10] Y.L. Kalinovsky, A.E. Radzhabov, and M. K. Volkov, hep-ph/0508264.

[11] C. P. Herzog, A. Karch, P. Kovtun, C. Kozcaz, and L. G. Yaffe, J. High Energy Phys. 07 (2006) 013.

[12] J. Casalderrey-Solana and D. Teaney, Phys. Rev. D 74, 085012 (2006).

[13] S. S. Gubser, hep-th/0605182.

[14] C. P. Herzog, J. High Energy Phys. 09 (2006) 032.

[15] E. Caceres and A. Guijosa, hep-th/0605235.

[16] J. J. Friess, S. S. Gubser, and G. Michalogiorgakis, J. High Energy Phys. 09 (2006) 072.

[17] S.-J. Sin and I. Zahed, hep-ph/0606049.

[18] F. Neri and A. Gocksch, Phys. Rev. D 28, 3147 (1983).

[19] R. D. Pisarski, Phys. Rev. D 29, 1222 (1984).

[20] S. A. Gottlieb et al., Phys. Rev. D 55, 6852 (1997).

[21] K. Ghoroku and M. Yahiro, Phys. Rev. D 73, 125010 (2006) .
[22] A. Bhattacharyya and S. Raha, J. Phys. G 21, 741 (1995).

[23] M. Asakawa and T. Hatsuda, Phys. Rev. Lett. 92, 012001 (2004).

[24] S. Datta, F. Karsch, P. Petreczky, and I. Wetzorke, Phys. Rev. D 69, 094507 (2004).

[25] S. Wissel, E. Laermann, S. Shcheredin, S. Datta, and F. Karsch, PoS LAT2005 (2006) 164 [hep-lat/0510031].

[26] H. Iida, T. Doi, N. Ishii, and H. Suganuma, PoS LAT2005 (2006) 184.

[27] R. Morrin et al., PoS LAT2005 (2006)176.

[28] M. Kruczenski, L. A.P. Zayas, J. Sonnenschein, and D. Vaman, J. High Energy Phys. 06 (2005) 046.

[29] S.-J. Rey, S. Theisen, and J.-T. Yee, Nucl. Phys. B527, 171 (1998).

[30] A. Brandhuber, N. Itzhaki, J. Sonnenschein, and S. Yankielowicz, Phys. Lett. B 434, 36 (1998).

[31] H. Liu, K. Rajagopal, and U. A. Wiedemann, hep-ph/ 0607062.

[32] M. Chernicoff, J. A. Garcia, and A. Guijosa, J. High Energy Phys. 09 (2006) 068.

[33] K. Peeters, J. Sonnenschein, and M. Zamaklar, J. High Energy Phys. 02 (2006) 009.

[34] K. Peeters, J. Plefka, and M. Zamaklar, J. High Energy Phys. 11 (2004) 054.

[35] K. Peeters, J. Plefka, and M. Zamaklar, Fortschr. Phys. 53, 640 (2005)

[36] E. Schreiber, hep-th/0403226.

[37] M. Shifman, hep-ph/0507246.

[38] A. Karch, E. Katz, D. T. Son, and M. A. Stephanov, Phys. Rev. D 74, 015005 (2006). 